\title{
PRODUÇÃO DE MUDAS DE MAMOEIRO UTILIZANDO BOKASHI E LITHOTHAMNIUM ${ }^{1}$
}

\author{
OSCAR MARIANO HAFLE² ${ }^{2}$, VERÔNICAANDRADE DOS SANTOS ${ }^{3}$, JOSÉ DARLAN RAMOS ${ }^{4}$, \\ MARIA DO CÉU MONTEIRO DA CRUZ ${ }^{5}$, PAULO CÉSAR DE MELO 6
}

RESUMO - O trabalho teve o objetivo avaliar o crescimento de mudas de mamoeiro do grupo Solo, sob diferentes doses dos fertilizantes naturais bokashi e pó de algas marinhas (Lithothamnium sp). O substrato utilizado foi a mistura de terra, areia e composto orgânico $(3: 2: 1, \mathrm{v} / \mathrm{v})$. Os tratamentos consistiram de quatro doses do fertilizante bokashi $\left(0 ; 3 ; 6 ; 10 \%\right.$, v/v) e quatro doses do fertilizante lithothamnium $\left(0 ; 3 ; 6 ; 10 \mathrm{~g} \mathrm{~L}^{-1}\right)$, adicionados ao substrato, antes do enchimento das sacolas. Utilizou-se o delineamento experimental em blocos inteiramente casualizados, em esquema fatorial $4 \mathrm{x} 4$, com quatro repetições e cinco plantas por parcela experimental. Foram realizadas avaliações aos 15; 30; 60 e 100 dias após a semeadura, sendo elas: emergência (\%), número de folhas, comprimento da parte aérea e da raiz $(\mathrm{cm})$, massa seca da parte aérea, da raiz e total $(\mathrm{mg})$. Houve interação significativa dos fatores testados para o comprimento da parte aérea, aos sessenta e cem dias após a semeadura; efeito isolado do bokashi para todas as variáveis analisadas, exceto na emergência, comprimento da parte aérea e número de folhas aos trinta dias após a semeadura. $\mathrm{O}$ uso conjugado dos fertilizantes mostrou efeito positivo na precocidade e altura da planta, podendo ser recomendado na formulação de substratos para a produção de mudas de mamoeiro do grupo Solo.

Termos para indexação: Carica papaya L., adubação orgânica, alga calcificada.

\section{PRODUCTION OF SEEDLINGS OF PAPAYA TREE USING BOKASHI AND LITHOTHAMNIUM}

\begin{abstract}
The work had the objective to evaluate the growth of papaya seedlings of the Solo group, under different doses of the natural fertilizers bokashi (fermented organic matter) and powderd sea algae (Lithothamnium $s p$ ). The substrate used was a mixture of soil, sand and organic compost $(3: 2: 1$, v/v). Treatments consisted of four doses of the bokashi fertilizer $(0 ; 3 ; 6 ; 10 \%, \mathrm{v} / \mathrm{v})$ and four doses of lithothamnium fertilizer $\left(0 ; 3 ; 6 ; 10 \mathrm{~g} \mathrm{~L}^{-1}\right)$, added to the substrate, before the filling of the bags. The experimental design used was completely randomized blocks, in a 4x4 factorial outline, with four repetitions and five plants per experimental plot. Evaluations were done at 15, 30, 60 and 100 days after seeding, which were: emergence $(\%)$, number of leaves, the shoot and the root length of the plant $(\mathrm{cm})$, aerial, root and total dry matter weight $(\mathrm{mg})$. There was significant interaction of the factors tested for the shoot length, at sixty and at hundred days after seeding; an isolated effect of the bokashi for all the analyzed variables, except for emergence, shoot length and number of leaves at thirty days after seeding. The conjugated use of the fertilizers showed a positive effect on precocity and plant height, and could be recommended in the formulation of substrate for the production of papaya seedlings.
\end{abstract}

Index terms: Carica papaya L., organic manuring, dried algae.

\footnotetext{
'(Trabalho 103-08). Recebido em: 24-04-2008. Aceito para publicação em: 13-01-2009.

${ }^{2}$ Doutor em Agronomia/Fitotecnia, Departamento de Agricultura/Universidade Federal de Lavras (DAG/UFLA). Cx. Postal 3037, Lavras, MG, CEP 37200-000; Professor no Instituto Federal de Educação, Ciência e Tecnologia da Paraíba, Campi de Sousa, PB omhafle@yahoo.com.br Autor correspondente.

${ }^{3}$ Doutoranda em Agronomia/Fitotecnia-DAG/UFLA-MG. veronicaandrad@yahoo.com.br

${ }^{4}$ Doutor Professor de Fruticultura -DAG/UFLA-MG. darlan@ufla.br

${ }_{5}^{5}$ Doutoranda em Agronomia/Fitotecnia-DAG/UFLA-MG. m_mariceu@yahoo.com.br

${ }^{6}$ Doutor Professor de Fruticultura -DAG/UFLA-MG. pcmelo2002@yahoo.com.br
} 


\section{INTRODUÇÃO}

O mamoeiro ocupa lugar de destaque na fruticultura nacional, sendo cultivado em diversas regiões do Brasil. As cultivares mais plantadas no Brasil pertencem aos grupos Solo e Formosa, sendo usadas nestes cultivos práticas convencionais, integradas e orgânicas.

A precocidade na produção de frutos do mamoeiro tem relação direta com o desenvolvimento inicial das mudas, sendo o padrão das mudas uma das características que interferem diretamente na produtividade do pomar (Trindade et al., 2000). Portanto, é necessário implantá-lo com mudas de alta qualidade, vigorosas e com estado nutricional adequado.

Para a produção de mudas, é importante a utilização de substratos que apresentem propriedades físico-químicas adequadas e que forneçam os nutrientes necessários para o bom desenvolvimento inicial da planta (Mendonça et al., 2002).

Um dos sistemas mais utilizados para a produção das mudas de mamoeiro é em sacos de polietileno preto $(18 \times 8 \mathrm{~cm})$, com substrato composto de uma mistura de subsolo peneirado, areia e esterco de curral bem curtido, na proporção de 3:1:1 (Costa et al., 2003). As recomendações de Costa \& Costa (2003) são para que, neste tipo de substrato, sejam incorporadas, por metro cúbico, as quantidades de 5 $\mathrm{kg}$ de superfosfato simples, $0,5 \mathrm{~kg}$ de cloreto de potássio e 1 a $2 \mathrm{~kg}$ de calcário.

Em substituição à adubação química, o fornecimento dos nutrientes para as plantas é feito, principalmente, por meio de adubações com materiais de origem orgânica, sendo bastante variados os tipos, formas de preparo, quantidade e disponibilidade dos nutrientes e nem sempre são suficientes para suprir a demanda nutricional, o que torna necessária a complementação com adubações minerais. É permitido, neste caso, o uso de alguns adubos minerais, a exemplo das cinzas, fosfatos de rochas, termofosfatos, carbonatos e pó de algas (Penteado, 2003).

O bokashi é um fertilizante orgânico, resultante de um método de compostagem baseado na adição de uma solução líquida de microrganismos efetivos, que são bactérias anaeróbicas e fermentos do ácido láctico (Souza e Resende, 2003). É um adubo orgânico concentrado, rico em nitrogênio, fósforo e potássio, para a substituição dos fertilizantes químicos tradicionais, podendo ser aplicado por ocasião do plantio ou em cobertura (Penteado, 2003).

De acordo com Souza \& Rezende (2003), a composição do bokashi pode variar de um preparo para outro, com valores nutricionais aproximadamente de: $\mathrm{N}=3 \% ; \mathrm{P}=2 \% ; \mathrm{K}=1,4 \% ; \mathrm{Ca}=2,2 \% ; \mathrm{Mg}=1,1 \%$; $\mathrm{Mn}=0,018 \% ; \mathrm{Zn}=0,011 \% ; \mathrm{Fe}=0,090 \% ; \mathrm{B}=0,020 \%$; $\mathrm{Cu}=0,010 \% ; \mathrm{pH}=6,0$ e relação $\mathrm{C} / \mathrm{N}=12: 1$ ). Segundo eles, o uso de doses crescentes destes fertilizantes pode afetar diretamente o acúmulo de massa seca, devido a maior disponibilidade de nutrientes no solo.

O lithothamnium é um material derivado de algas marinhas calcárias, pertencente à família Corallinales, do gênero Lithothamnium, sendo já relatadas mais de 25 espécies (wikipedia, 2008). Segundo Melo \& Furtini Neto (2003), o produto apresentou as seguintes características químicas: 462,7 g.kg ${ }^{-1}$ de $\mathrm{CaO} ; 330,5$ g.kg-1 de Ca; 42,3 g. $\mathrm{kg}^{-1}$ de $\mathrm{MgO} ; 25,4$ g. $\mathrm{kg}^{-1}$ de $\mathrm{Mg}$; reatividade $(99,26 \%)$ e PRNT $(92,62 \%)$. O produto foi eficiente como corretivo da acidez do solo, proporcionando melhorias significativas no crescimento e na produção do feijoeiro, sendo a dose de melhor resposta entre 0,5 a 1 tonelada por hectare.

O elevado custo dos substratos comerciais tem levado à utilização de outros materiais disponíveis na propriedade (terra, areia e compostos orgânicos), principalmente entre os pequenos produtores rurais, os quais produzem as suas próprias mudas, para diversas espécies vegetais de propagação relativamente mais simples, a exemplo do mamão.

Portanto, objetivou-se neste trabalho avaliar o crescimento inicial das plantas de mamoeiro do grupo Solo, sob diferentes doses dos fertilizantes bokashi e lithothamnium, sob condição de casa de vegetação, em Lavras-MG.

\section{MATERIAL E MÉTODOS}

O experimento foi instalado e conduzido em casa de vegetação, no Setor de Fruticultura da Universidade Federal de Lavras (UFLA), em LavrasMG, no período de março a junho de 2007.

Como material propagativo, foram usadas sementes de mamoeiro (Carica papaya L.), do grupo Solo, semeadas, duas em cada sacola de polietileno preto $(0,6 \mathrm{~L})$, contendo os substratos e mantidas sob ambiente protegido, por um período de 100 dias.

O substrato-padrão utilizado foi uma mistura de terra de barranco, areia de textura média e composto orgânico $(3: 2: 1, \mathrm{v} / \mathrm{v})$, tendo as seguintes características: $\mathrm{pH}\left(\mathrm{H}_{2} \mathrm{O}\right)=6,5 ; \mathrm{P}($ Mehlich $)=30,8$ mg.dm ${ }^{-3} ; \mathrm{K}=144 \mathrm{mg} . \mathrm{dm}^{-3} ; \mathrm{Ca}^{2+}=3,1 \mathrm{cmolc} . \mathrm{dm}^{-3}$; $\mathrm{Mg}^{2+}=1,2$ cmolc. $\mathrm{dm}^{-3} ; \mathrm{Al}^{3+}=0,0$ cmolc. $\mathrm{dm}^{-3}$; $\mathrm{H}+\mathrm{Al}^{3+}=1,8$ cmolc. $\mathrm{dm}^{-3} ; \mathrm{SB}=5,7 \mathrm{mg} \cdot \mathrm{dm}^{-3} ; \mathrm{T}=6,9$ 
mg.dm ${ }^{-3} ; \mathrm{V}=82,6 \%$; cmolc. $\mathrm{dm}^{-3} ; \mathrm{MO}=2,2$ dag. $\mathrm{dm}^{-3}$; Areia $=37$ dag. $\mathrm{dm}^{-3} ;$ Silte $=18$ dag. $\mathrm{dm}^{-3} ;$ Argila $=45$ dag. $\mathrm{dm}^{-3}$.

Os tratamentos consistiram de quatro doses do fertilizante bokashi, na forma farelada $(0 ; 3 ; 6 ; 10$ $\%, \mathrm{v} / \mathrm{v})$ e quatro doses do fertilizante lithothamnium, na forma de pó $\left(0 ; 3 ; 6 ; 10 \mathrm{~g} \mathrm{~L}^{-1}\right)$, adicionados ao substrato antes do enchimento das sacolas plásticas. Foi utilizado o delineamento experimental em blocos inteiramente casualizados, no esquema fatorial 4 x 4, com quatro repetições e cinco plantas por parcela experimental.

Quinze dias após o início da emergência, foi realizada a avaliação deste percentual e, em seguida, o desbaste cortando as plantas com tesoura, deixando apenas a planta mais vigorosa por sacola. As irrigações foram realizadas diariamente, através de regador manual, mantendo o substrato sempre próximo à capacidade de campo.

Aos trinta, sessenta e cem dias após a semeadura, foram realizadas as avaliações do número de folhas e comprimento da parte aérea, sendo que, aos cem dias, foram incluídas as medições de comprimento da raiz, massa seca da parte aérea, da raiz e total.

O percentual de emergência foi calculado dividindo-se o número de plantas que emergiram pelo número de sementes semeadas e multiplicando por 100. O número de folhas foi avaliado mediante a contagem de todas as folhas da planta. O comprimento da parte aérea foi determinado pela medida desde o colo até a gema terminal da planta, e o comprimento da raiz, desde o colo até a ponta da raiz mais comprida, utilizando régua graduada em milímetros. A massa seca da parte aérea, da raiz e total foi verificada após a secagem do material em estufa de circulação forçada por um período de 72 horas, sendo os resultados expressos em miligramas.

Os dados obtidos foram submetidos à análise de variância, ao nível de significância de $5 \%$. Quando a interação entre bokashi e lithothamnium se mostrou significativa $(p<0,05)$, procedeu-se ao desdobramento dos fatores. Foi realizada a análise de regressão, sendo determinadas as equações linear e quadrática, utilizando o aplicativo SISVAR (Ferreira, 2000).

\section{RESULTADOS E DISCUSSÃO}

Não foi observada influência dos produtos nas variáveis: emergência, comprimento da parte aérea e número de folhas aos trinta dias após a semeadura (Tabela 1). Houve efeito da utilização do bokashi para todas as variáveis analisadas e do pó de algas marinhas (lithothamnium) apenas na variável comprimento da parte aérea, aos cem dias. Verificouse interação significativa entre os produtos testados (bokashi x lithothamnium) para a variável comprimento da parte aérea, aos sessenta e cem dias após a semeadura.

$\mathrm{Na}$ avaliação do comprimento da parte aérea realizada aos sessenta dias, observou-se que a incorporação do bokahi e lithothamnium ao substrato favoreceu o crescimento linear das mudas (Figura 1). Para as mudas que não receberam a aplicação do lithothamnium, a incorporação de apenas bokashi proporcionou comportamento quadrático, sendo que o maior comprimento da parte aérea $(8,06 \mathrm{~cm})$ deu-se na dose de 7,19 \% do bokashi no substrato-padrão. O aumento da quantidade de bokashi reduziu o comprimento médio da planta. Para os tratamentos com a incorporação do lithothamnium, o comportamento foi linear, com acréscimos de 74,7\%, $39,3 \%$ e $39,8 \%$, tendo sido a dose de 3 g. $\mathrm{L}^{-1}$ mais eficiente entre as doses testadas do produto.

$\mathrm{Na}$ avaliação realizada aos cem dias, o comprimento da parte aérea apresentou comportamento quadrático para todas as doses do lithothamnium testadas (Figura 2). Os maiores comprimentos foram de 27,73; 30,71; 30,29 e 27,82 cm, respectivamente, para as doses de 7,69; 5,72; 5,53 e $6,49 \%$ de bokashi e $0 ; 3 ; 6$ e $10 \mathrm{~g} \mathrm{~L}^{-1}$ do lithothamnium. Esses resultados sugerem que o lithothamnium interage, exercendo uma ação corretiva da acidez do substrato, conforme relatado por Melo \& Furtini Neto (2003), favorecendo o crescimento das mudas nas doses mais altas do bokashi.

Na Figura 3, observa-se que a equação de regressão mostrou comportamento linear em relação às doses do fertilizante bokashi, mostrando que o aumento da dose deste proporcionou o crescimento linear do número de folhas aos sessenta dias, em qualquer que seja a dose do lithothamnium. No entanto, aos cem dias, esta mesma variável apresentou um comportamento quadrático, com o máximo de 14,95 folhas por planta, estimado com concentração de $6,13 \%$ de bokashi adicionada ao substrato (Figura 4).

O aumento da área foliar foi possível devido ao efeito físico da matéria orgânica no substrato, pois segundo Kiehl (1985) ela melhora a estrutura do solo, reduz a plasticidade e a coesão, aumenta a capacidade de retenção de água e a aeração, permitindo maior penetração e distribuição das raízes. Estas condições permitiram que as plantas mantivessem, na parte aérea, o maior número de folhas aos sessenta dias; no entanto, esta mesma variável, analisada aos cem dias após a semeadura, mostrou que o incremento do 
fertilizante bokashi, acima das doses ótimas, proporcionou decréscimo na emissão de folhas. Este fato pode estar associado ao decréscimo do efeito neutralizante da acidez neste tempo de avaliação, conforme discutido anteriormente.

O comprimento da raiz, aos cem dias, mostrou comportamento linear em relação às doses do fertilizante bokashi (Figura 5). A massa seca da parte aérea, da raiz e total, realizada nesta mesma época, mostrou comportamento quadrático para todas as doses do lithothamnium, mas sem interação significativa, com relação às doses do fertilizante bokashi. Os maiores valores calculados foram de $2.176,8 ; 1.307,7$ e $868,9 \mathrm{mg}$, respectivamente, para as doses de 7,41; 7,47 e 7,44\% de bokashi no substrato
(Figura 6).

Resultados semelhantes foram encontrados por Souza et al. (2007), quando testaram diferentes doses do pó de algas ( 0 a $\left.10 \mathrm{~kg} . \mathrm{m}^{-3}\right)$, não encontrando efeitos significativos das doses do produto no número de folhas, comprimento da raiz e matéria seca da parte aérea e da raiz nas plantas de maracujazeirodoce, na fase de formação das mudas.

Conforme apresentado por Souza \& Rezende (2003), a composição do bokashi traz valores consideráveis de elementos nutritivos para as plantas, porém possui $\mathrm{pH}$ levemente ácido $(6,0) \mathrm{e}$ alta relação $\mathrm{C} / \mathrm{N}$ (12:1). O uso de doses muito elevadas deste composto pode acidificar o solo e até mesmo consumir nitrogênio durante sua decomposição.

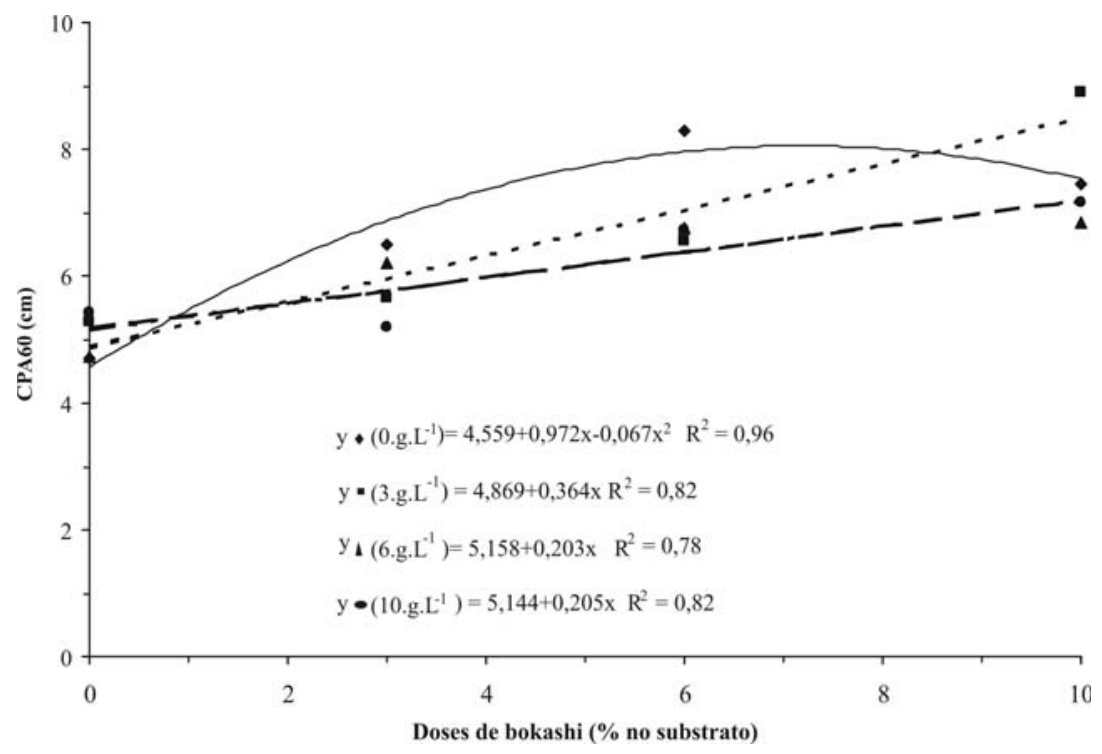

FIGURA 1 - Comprimento da parte aérea avaliado aos 60 dias (CPA60), nas mudas de mamoeiro, em função das diferentes doses de bokashi e lithothamnium. UFLA, Lavras-MG, 2007.

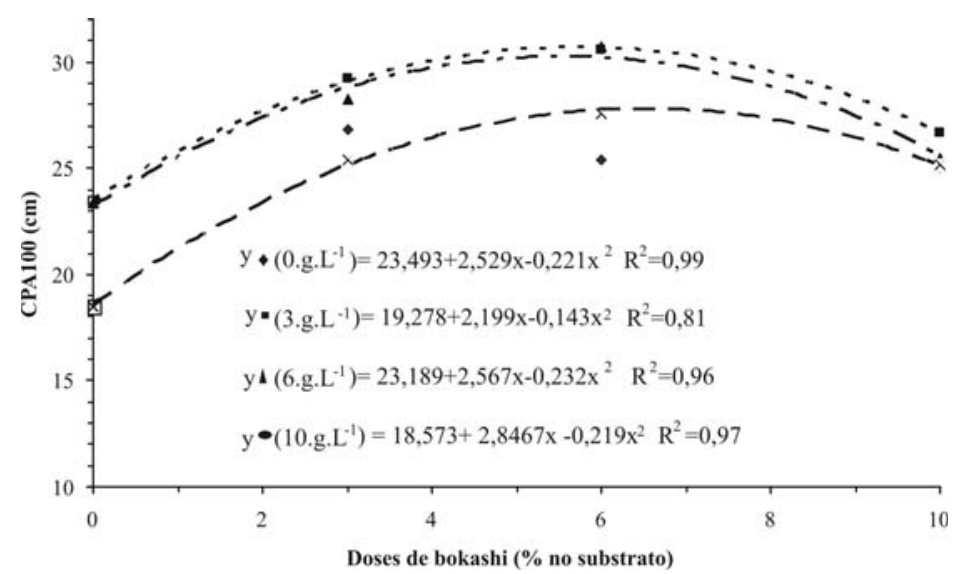

FIGURA 2 - Comprimento da parte aérea avaliado aos 100 dias (CPA100), nas mudas de mamoeiro, crescidas em diferentes doses de bokashi e lithothamnium. UFLA, Lavras-MG, 2007. 


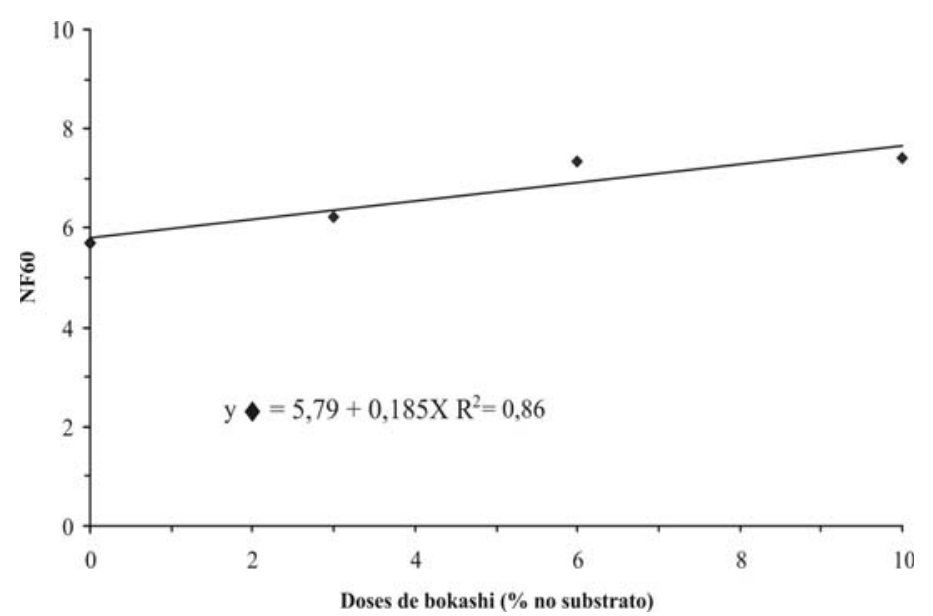

FIGURA 3 - Número de folhas avaliado aos 60 dias (NF60), nas mudas de mamoeiro, crescidas em diferentes doses de bokashi e lithothamnium. UFLA, Lavras-MG, 2007.

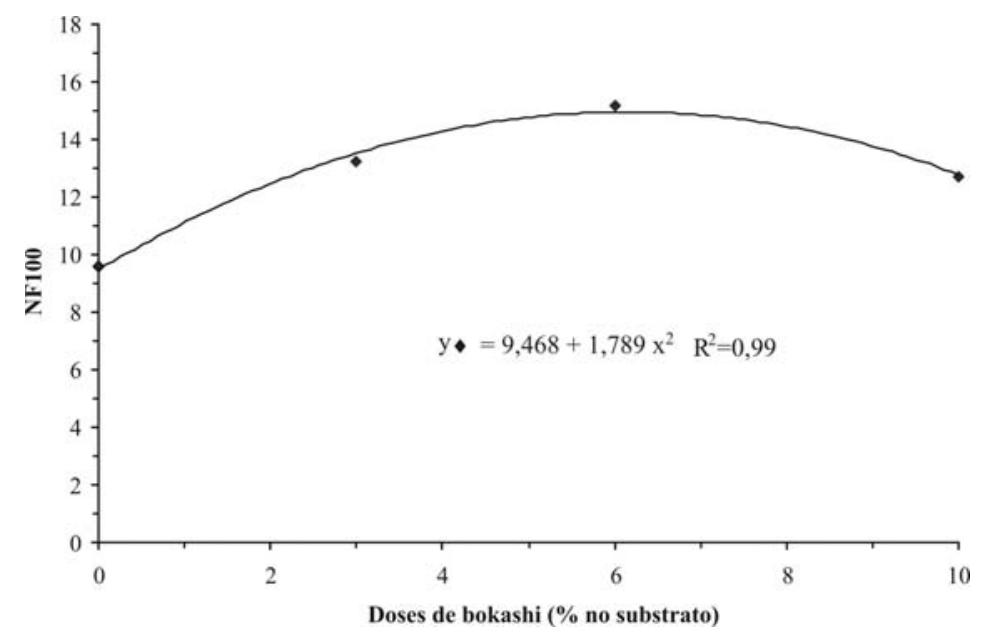

FIGURA 4 - Número de folhas avaliado aos 100 dias (NF100), nas mudas de mamoeiro, crescidas em diferentes doses de bokashi e lithothamnium. UFLA, Lavras-MG, 2007.

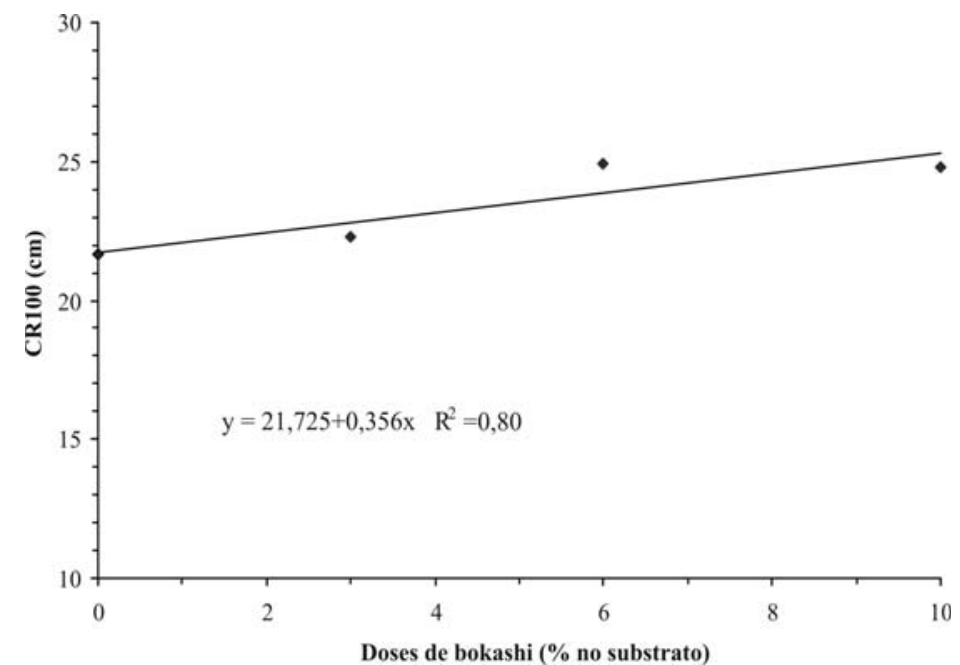

FIGURA 5 - Comprimento da raiz (CR100) aos 100 dias, nas mudas de mamoeiro, crescidas em diferentes doses de bokashi e lithothamnium. UFLA, Lavras-MG, 2007. 


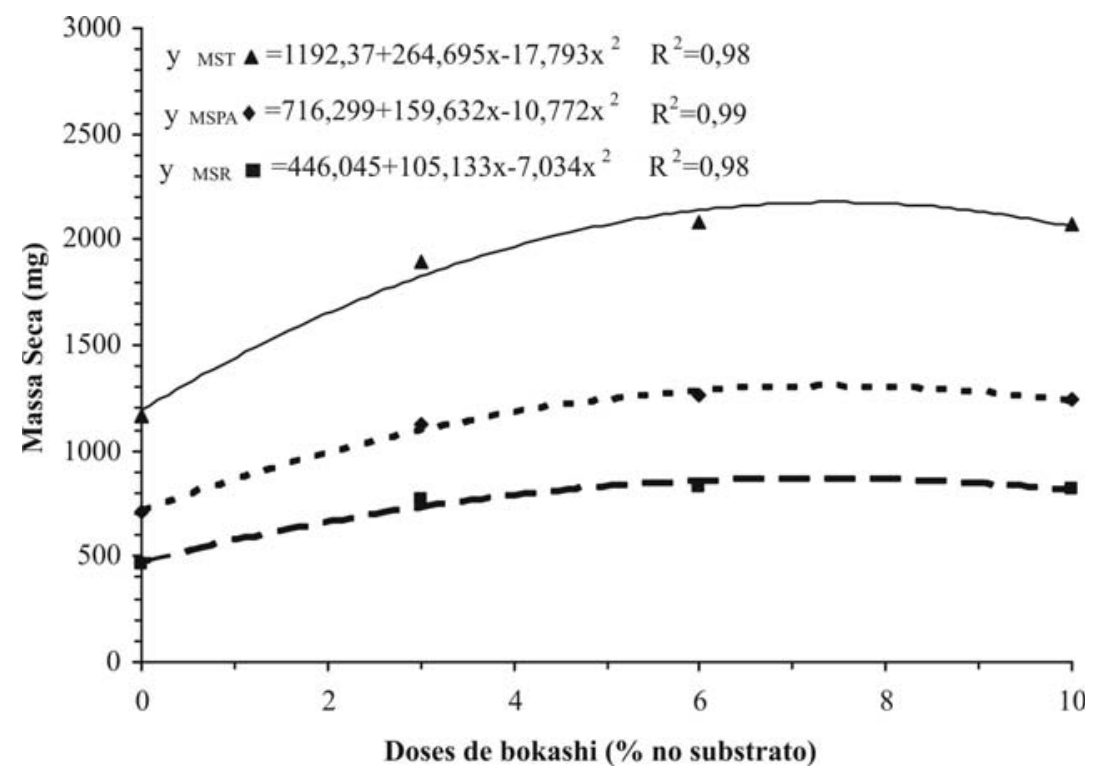

FIGURA 6 - Massa seca da parte aérea (MSPA), da raiz (MSR) e total (MST) aos 100 dias, nas mudas de mamoeiro, crescidas em diferentes doses de bokashi e lithothamnium. UFLA, Lavras-MG, 2007.

TABELA 1 - Quadrado médio, média e coeficiente de variação, das características de comprimento da parte aérea (CPA60) e número de folhas (NF60) aos sessenta dias; comprimento da parte aérea (CPA100), número de folhas (NF100) e comprimento da raiz (CR100) aos 100 dias: massa seca da parte aérea (MSPA), da raiz (MSR) e total (MST) das plantas de mamoeiro.UFLA, LavrasMG, 2007.

\begin{tabular}{cccccccccc}
\hline $\begin{array}{c}\text { Fonte de } \\
\text { Variação }\end{array}$ & GL & $\begin{array}{c}\text { CPA60 } \\
(\mathbf{c m})\end{array}$ & NF60 & $\begin{array}{c}\text { CPA100 } \\
(\mathbf{c m})\end{array}$ & NF100 & $\begin{array}{c}\text { CR100 } \\
(\mathbf{c m})\end{array}$ & $\begin{array}{c}\text { MSPA } \\
(\mathbf{m g})\end{array}$ & $\begin{array}{c}\text { MSR } \\
(\mathbf{m g})\end{array}$ & $\begin{array}{c}\text { MST } \\
(\mathbf{m g})\end{array}$ \\
\hline & & & & & & & & & \\
Bloco & 3 & $1,525^{\mathrm{ns}}$ & $0,476^{\mathrm{ns}}$ & $4,082^{\mathrm{ns}}$ & $17,692^{\mathrm{ns}}$ & $1,355^{\mathrm{ns}}$ & $33797^{\mathrm{ns}}$ & $64837^{\mathrm{ns}}$ & $182359^{\mathrm{ns}}$ \\
Bokashi (B) & 3 & $21,449^{* *}$ & $11,586^{* *}$ & $96,641^{* *}$ & $87,139^{* *}$ & $46,172^{* *}$ & $1072411^{* *}$ & $485523^{* *}$ & $2998026^{* *}$ \\
Lithothamnium (L) & 3 & $1,609^{\mathrm{ns}}$ & $0,596^{\mathrm{ns}}$ & $31,731^{*}$ & $8,559^{\mathrm{ns}}$ & $1,288^{\mathrm{ns}}$ & $43323^{\mathrm{ns}}$ & $16038^{\mathrm{ns}}$ & $105470^{\mathrm{ns}}$ \\
B x L & 9 & $2,062^{*}$ & $0,368^{\mathrm{ns}}$ & $22,757^{*}$ & $8,573^{\mathrm{ns}}$ & $8,469^{\mathrm{ns}}$ & $97678^{\mathrm{ns}}$ & $73626^{\mathrm{ns}}$ & $307549^{\mathrm{ns}}$ \\
Resíduo & 45 & 0,764 & 0,585 & 8,672 & 5,290 & 9,987 & 78399 & 37124 & 202539 \\
\hline Média & - & 6,39 & 6,67 & 26,04 & 12,68 & 23,42 & 1084,08 & 720,43 & 1804,67 \\
CV (\%) & - & 13,67 & 11,46 & 11,31 & 18,14 & 13,49 & 25,83 & 26,74 & 24,94 \\
\hline
\end{tabular}

Teste $\mathrm{F} * *$ significativo $(\mathrm{P}<0,01)$, *significativo $(\mathrm{P}<0,05)$, ${ }^{\text {ns }}$ não significativo 


\section{CONCLUSÕES}

1-A adição do fertilizante bokashi no substrato, em doses de 6 a $7 \%(\mathrm{v} / \mathrm{v})$, promove o aumento do número de folhas, comprimento da parte aérea e massa seca da parte aérea, da raiz e total, enquanto doses próximas a $10 \%$ favorecem o crescimento da raiz.

2-O fertilizante natural lithothamnium apresentou melhores respostas na dose de 3 g. $\mathrm{L}^{-1}$, para as variáveis analisadas.

3-O uso conjugado dos fertilizantes mostrou efeito positivo, principalmente na altura da planta, característica muito importante na avaliação da qualidade de mudas.

\section{REFERÊNCIAS}

COSTA, A. de F.S. da; COSTA, A.N.da; SANTOS, F.A.M. dos; BARRETO, F.C.; ZUFFO, V.J. (eds.). Plantio, formação e manejo da cultura. In: MARTINS, D. dos S.; COSTA, A. de F.S. da. A cultura do mamoeiro: tecnologias de produção. Vitória: INCAPER, 2003. p. 127-159.

COSTA, A.N.da; COSTA, A. de F.S. da. Nutrição e adubação. In: MARTINS, D. dos S.; COSTA, A. de F.S. da. (eds.).A cultura do mamoeiro: tecnologias de produção. Vitória: INCAPER, 2003. p. 201-227.

FERREIRA, D.F. Análise estatística por meio do SISVAR (Sistema para Análise de Variância) para Windows, versão 4.0. In: REUNIÃO ANUAL DA REGIÃO BRASILEIRA DA SOCIEDADE INTERNACIONAL DE BIOMETRIA, 45., 2000, São Carlos. Anais... São Carlos: UFSCar, 2000. p.255-258.
KIEHL, J.E. Fertilizantes orgânicos. Piracicaba: Agronômica Ceres, 1985. 492 p.

MELO, P.C.; FURTINI NETO, A.E. Avaliação do lithothamnium como corretivo da acidez do solo e fonte de nutrientes para o feijoeiro. Revista Ciência eAgrotecnologia, Lavras, v.27, n.03, p.508-519, 2003.

MENDONÇA, V.; RAMOS, J. D.; ARAÚJO NETO, S. E. de; PIO, R.; GONTIJO, T. C. A.; JUNQUEIRA, K. P. Substratos e quebra de dormência na formação do porta-enxerto de gravioleira cv. RBR. Revista Ceres, Viçosa, v. 49, n. 286, p. 657-668, 2002.

PENTEADO, S.R. Introdução à agricultura orgânica. Viçosa: Grafica Impress, 2003. 235p.

SOUZA, H.A. de; MENDONÇA, V.; RAMOS, J.D.; FERREIRA, E.A.; ALENCAR, R.D. Doses de lithothamnium e diferentes substratos na produção de mudas de maracujazeiro-doce. Revista Caatinga, Mossoró, v.20, n.4, p.24-30, 2007.

SOUZA, J.L.; RESENDE, P. Manual de horticultura orgânica. Viçosa: Aprenda Fácil, 2003. 564p.

TRINDADE, A.V.; FARIA, N.G.; ALMEIDA, F.P. Uso de esterco no desenvolvimento de mudas de mamoeiro colonizadas com fungos micorrízicos. Pesquisa Agropecuária Brasileira, Brasília, v.35, n.7, 2000.

WIKIPEDIA 2008. Disponível em: <http:// fr.wikipedia.org/wiki/Lithothamnium >. Acesso em: 15 mar. 2008. 